\title{
Benefits of Position-Sensitive Detectors for Source Detection with Known Background
}

\author{
Daniel J. Lingenfelter, Jeffrey A. Fessler, Clayton D. Scott, and Zhong He
}

\begin{abstract}
We address the question of whether or not the directional or imaging information offered by a position-sensitive gamma-ray detector improves the detection accuracy when searching for a source of known shape amid a background of known intensity. We formulate the detection problem as a composite hypothesis testing problem and examine the behavior of the generalized likelihood ratio test (GLRT) in terms of the area under the receiver operating characteristic (AUC). Due to the analytical complexity of the GLRT in this case, we examine its asymptotic properties when the number of detected photons is large. We find that a detector of uniform sensitivity can more accurately detect a source when imaging information is used.
\end{abstract}

\section{INTRODUCTION}

One means of detecting radioactive material is to count the gamma-ray photons emitted from it. Such a photon-counting detector, hereafter referred to as a non-imaging detector, is limited by its inability to obtain information about the direction of incoming photons. It is reasonable to hypothesize that directional information can improve detection capability by distinguishing between photons from a localized source and photons from a distributed background. A common assumption in the field of security imaging is that sources of radiation are small, so they appear point-like to a detector [1]. In light of this, we consider the problem of detecting a point-source in a known diffuse background. In this work, we examine the detection performance using the generalized likelihood ratio test (GLRT).

When performing detection using the GLRT with a position-sensitive Compton imaging detector in a known background, a particular experiment found that imaging information did not significantly improve detection performance over merely counting received photons [2]. In light of the numerous modalities that contain some form of imaging information, we seek to show theoretically how the information added by imaging capability affects detection performance. The goals of this work are to explain previous empirical results, such as those contained in [2], and guide the design of future detectors.

The question of whether or not imaging information improves detection performance was addressed in [3] in the context of coded-aperture imaging systems. If the background intensity is known, reference [3] argued that imaging information does not improve the signal-to-noise ratio (SNR), therefore imaging information does not improve detection performance. However, if the background intensity is unknown, imaging may improve SNR [3] and thus detection

This work was supported by DNDO of DHS grant number 2008-DN-077ARI007-02. performance by providing a means to separate the otherwise indistinguishable source and background photons. The analysis in this paper differs from that of [3] because we treat the problem from a detection task-based point of view. We analyze the task of source detection using the asymptotic performance of the GLRT applied to photon counting imaging and nonimaging detectors. To the best of our knowledge, there is no other literature analyzing the impact of imaging capability on detection performance for photon-counting detectors.

In this work, we quantify detection performance using the area under the receiver operating characteristic (AUC). This metric is independent of any particular threshold value and is a measure of the overall detectability [4].

The novel contribution of this work is the task-based analysis of the asymptotic performance of the GLRT for detecting a single source in background for photon counting detectors with and without imaging capability. We give Theorem 1, which states that in a known background, a uniform-sensitivity detector with imaging capability always has better detection performance asymptotically in terms of AUC than a nonimaging detector of equal sensitivity. This theorem, to our knowledge, is the first to answer the question of whether or not imaging capability improves detection performance from the task-based point of view of the GLRT when the background is known. Our analysis also provides an expression that quantifies how much imaging capability increases the AUC.

\section{MAthematicAl BACKGROUND}

The model described in this section is general enough to describe any system that records a Poisson-distributed number of events, where the events are independent and can be described by a vector of attributes. This model accurately describes position-sensitive Compton detectors, coded aperture detectors, and scintillator arrays.

\section{A. Measurement Model}

Let $\tilde{\boldsymbol{r}}=\left(\boldsymbol{r}_{1}, \boldsymbol{r}_{2}, \ldots, \boldsymbol{r}_{J}\right)$ be a vector of recorded attributes of photon interaction events. Each element $\boldsymbol{r}_{i}$ of $\tilde{\boldsymbol{r}}$ is itself a vector of attributes describing the $i$ th event. An example of a detector that one can describe with this model is a positionsensitive Compton detector. A Compton detector records a Poisson-distributed number $J$ of gamma-ray photons. Each detected photon interacts one or more times inside the detector and the detector records these interaction locations. For this system, the vector $\boldsymbol{r}_{i}$ is populated with the interaction locations of the $i$ th recorded photon. The assumption that the attributes 
of recorded events are independent is reasonable provided the count rates are low enough to avoid the dead time effect [5].

In the detection problem considered here, the goal is to decide whether or not a point-source is present in an environment with some background. The two parameters characterizing the source are the intensity $\alpha \in[0, \infty)$ having units of emissions per unit time and source position $\phi \in \Phi$. In the 3D far-field, an example for the set $\Phi$ is $[0,2 \pi) \times[0, \pi]$, which represents all possible azimuth and polar angles on a sphere. Since we are only considering uniform-sensitivity detectors, the system sensitivity is independent of source position and we denote it by $s_{0}$. The mean number of background counts is denoted by $\lambda_{b}$ and is assumed known. We define the vector $\boldsymbol{\theta}$ to be the vector of all unknown parameters,

$$
\boldsymbol{\theta}=(\alpha, \boldsymbol{\phi}),
$$

and $D$ to be the event that a photon is recorded.

Let $\mathrm{p}(\boldsymbol{r} \mid D ; \boldsymbol{\theta})$ denote the distribution of recorded attributes $r \in \mathcal{R}$, where $\mathcal{R}$ is the set of all possible event attributes, parameterized by $\boldsymbol{\theta}$. To characterize imaging capability, we introduce two probability distributions: $\mathrm{p}_{S}(\boldsymbol{r} \mid D ; \boldsymbol{\phi})$ and $\mathrm{p}_{B}(\boldsymbol{r} \mid D)$, which are the distributions of recorded event attributes $r$ given that they are detected and come from the source and background, respectively. The overall distribution of recorded attributes (given that an event is detected) is $\mathrm{p}(\boldsymbol{r} \mid D ; \boldsymbol{\theta})$, and it is a mixture of $\mathrm{p}_{S}(\boldsymbol{r} \mid D ; \phi)$ and $\mathrm{p}_{B}(\boldsymbol{r} \mid D)$ given by

$$
\mathrm{p}(\boldsymbol{r} \mid D ; \boldsymbol{\theta})=\frac{\lambda_{b} \mathrm{p}_{B}(\boldsymbol{r} \mid D)+\alpha s_{0} \mathrm{p}_{S}(\boldsymbol{r} \mid D ; \boldsymbol{\phi})}{\lambda_{b}+\alpha s_{0}} .
$$

As shown in [5], the likelihood of $\boldsymbol{\theta}$ is

$$
\mathrm{p}(\tilde{\boldsymbol{r}} ; \boldsymbol{\theta})=\prod_{i=1}^{J} \mathrm{p}\left(\boldsymbol{r}_{i} \mid D ; \boldsymbol{\theta}\right) e^{-\bar{J}(\boldsymbol{\theta})} \bar{J}(\boldsymbol{\theta})^{J} / J !,
$$

and the number of recorded photons obeys the Poisson distribution

$$
J \sim \operatorname{Poisson}(\bar{J}(\boldsymbol{\theta})),
$$

with mean given by

$$
\bar{J}(\boldsymbol{\theta}) \triangleq \mathrm{E}[J]=\tau\left(\lambda_{b}+\alpha s_{0}\right),
$$

where $\tau$ denotes the known scan time.

We can make the concept of an imaging detector more concrete by the following definition:

Definition 1. A detector is a non-imaging detector if and only if $\mathrm{p}_{S}(\boldsymbol{r} \mid D ; \boldsymbol{\phi})=\mathrm{p}_{B}(\boldsymbol{r} \mid D)$ almost everywhere ${ }^{1}$ for all $\boldsymbol{\phi} \in \Phi$.

Definition 1 says that in a non-imaging detector, the distribution of event attributes is independent of whether or not the event originated from the source. Otherwise we call it an imaging detector. Note that with this definition of a nonimaging detector, the source and background spectra are either the same or at least have the same effect on the distribution of the recorded attributes.

\footnotetext{
${ }^{1}$ Throughout, "almost everywhere" means with respect to the distribution of $\boldsymbol{r}$ in (2)
}

\section{B. Generalized Likelihood Ratio Test (GLRT)}

In the source detection problem, we would like to discern between two hypotheses:

$$
\begin{aligned}
& H_{0}: \quad \alpha=0, \\
& H_{1}: \quad \alpha>0 .
\end{aligned}
$$

The GLRT is a common method of choosing between two hypotheses when one or more of the hypotheses depend on unknown parameters [6]. We can write the GLRT as

$$
2 \log \Lambda \underset{H_{0}}{\stackrel{H_{1}}{\gtrless} \gamma,}
$$

where the test statistic is

$$
\Lambda \triangleq \frac{\max _{\alpha, \phi} \mathrm{p}\left(\tilde{\boldsymbol{r}} ;(\alpha, \phi), H_{1}\right)}{\max _{\boldsymbol{\phi}} \mathrm{p}\left(\tilde{\boldsymbol{r}} ;(\alpha=0, \phi), H_{0}\right)} .
$$

To calibrate the test threshold $\gamma$ and analyze the performance of the detector, one must determine the distribution of $\Lambda$. In the case of (5), the distribution of $\Lambda$ is complicated because under $H_{0}$, the parameter $\alpha$ lies on the boundary of the parameter space [7]. To simplify the analysis and give intuition, instead of (5), we consider the two-sided test

$$
\begin{aligned}
& H_{0}: \quad \alpha=0 \\
& H_{1}: \quad \alpha \neq 0,
\end{aligned}
$$

which is also the basis of the analysis in [8]. When the test is treated with the two-sided formulation in (7), it is shown in [6, pp. 239-240] that the asymptotic distribution of the test statistic is given by

$$
2 \log (\Lambda) \sim \begin{cases}\chi_{1}^{2}(\eta) & H_{1} \\ \chi_{1}^{2}(0) & H_{0}\end{cases}
$$

where $\chi_{1}^{2}(\eta)$ denotes the non-central chi-square distribution with one degree of freedom and noncentrality parameter $\eta$. For the model (3), the noncentrality parameter is

$$
\eta=\alpha^{2}\left(\mathrm{~F}^{-1}(\boldsymbol{\theta})_{[1,1]}\right)^{-1}
$$

where $F(\boldsymbol{\theta})$ is the Fisher Information Matrix. The next section uses (8) to determine how imaging information affects detection performance.

\section{EFFeCt of IMAging ON Detection PeRformance}

As illustrated in Figure 1, the AUC of the GLRT for (7) is a monotone function of the noncentrality parameter $\eta$ in (8), so showing that imaging capability improves AUC is equivalent to showing that imaging capability increases $\eta$. We derive the Fisher information for an imaging detector and show that imaging always improves detection performance by increasing the AUC when detector sensitivity is uniform.

The noncentrality parameter in (8) depends only on the $[1,1]$ component of the inverse of $F(\boldsymbol{\theta})$. To facilitate the analysis of $F(\boldsymbol{\theta})$, we define the block components of the matrix as follows:

$$
\mathrm{F}(\boldsymbol{\theta})=\left[\begin{array}{ll}
\mathrm{F}_{[1,1]} & \mathrm{F}_{[2,1]}^{T} \\
\mathrm{~F}_{[2,1]} & \mathrm{F}_{[2,2]}
\end{array}\right],
$$




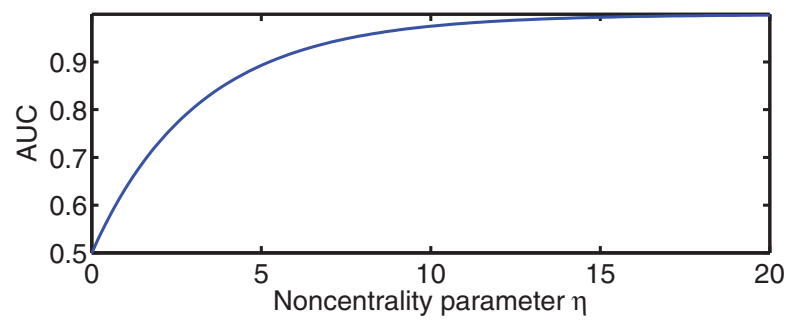

Fig. 1: AUC of GLRT (7) versus noncentraltity parameter $\eta$ of the asymptotic distribution of the GLRT.

where $F_{[1,1]}$ is $1 \times 1, F_{[2,1]}$ is $\operatorname{dim}(\phi) \times 1$, and $F_{[2,2]}$ is $\operatorname{dim}(\phi) \times$ $\operatorname{dim}(\phi)$.

\section{A. Fisher Information Expressions}

A non-imaging detector is neither capable of estimating $\phi$ nor distinguishing source and background events of the same energy because it does not record any position information, so the Fisher information is a scalar in this case. Using the model in (3) and (4), one can show that the log-likelihood for the non-imaging case is

$$
\mathbf{L}(\boldsymbol{\theta})=J \log \left(\tau\left(\lambda_{b}+\alpha s_{0}\right)\right)-\tau\left(\lambda_{b}+\alpha s_{0}\right),
$$

for which the Fisher information is given by

$$
\tilde{\mathrm{F}}(\boldsymbol{\theta}) \triangleq \frac{\tau s_{0}^{2}}{\lambda_{b}+\alpha s_{0}} .
$$

Note that increasing the scan time $\tau$ increases the Fisher information, whereas increasing the background rate $\lambda_{b}$ decreases the Fisher information, consistent with intuition. It is somewhat counter-intuitive that the Fisher information decreases as source intensity $\alpha$ increases, but the AUC still increases as a function of $\alpha$ because of the $\alpha^{2}$ term in (8).

To help express the Fisher information matrix (9) for an imaging detector, we first define the following functions of $r$ :

$$
\begin{aligned}
& g_{1}(\boldsymbol{r})=\mathrm{p}_{S}(\boldsymbol{r} \mid D ; \boldsymbol{\phi}) \\
& g_{2}(\boldsymbol{r})=\nabla_{\boldsymbol{\phi}}\left(s_{0} \mathrm{p}_{S}(\boldsymbol{r} \mid D ; \boldsymbol{\phi})\right) .
\end{aligned}
$$

Note that $g_{1}: \mathcal{R} \rightarrow \mathbb{R}$, and $g_{2}: \mathcal{R} \rightarrow \mathbb{R}^{\operatorname{dim}(\phi)}$. Using (2), (3), and (4), one can show that the Fisher Information for an imaging detector is given by

$$
\mathrm{F}(\boldsymbol{\theta})=\tilde{\mathrm{F}}(\boldsymbol{\theta})\left[\begin{array}{cc}
K_{[1,1]} & \frac{\alpha K_{[2,1]}^{T}}{s_{0}} \\
\frac{\alpha K_{[2,1]}}{s_{0}} & \frac{\alpha^{2} K_{[2,2]}}{s_{0}^{2}}
\end{array}\right],
$$

where

$$
K_{[i, j]} \triangleq \mathrm{E}\left[\frac{g_{i}(\boldsymbol{r}) g_{j}^{T}(\boldsymbol{r})}{\mathrm{p}(\boldsymbol{r} \mid D ; \boldsymbol{\theta})^{2}}\right],
$$

provided that the expectation integral and the gradient with respect to the parameters are interchangeable.

\section{B. Effect of Imaging Capability for a Uniform Sensitivity Detector with Known Background}

The main result of this section, given by Theorem 1, is that the detection performance of a uniform-sensitivity imaging detector is greater than that of a uniform sensitivity detector without imaging capability. The AUC of an imaging detector is greater than that of a non-imaging detector if the noncentrality parameter of the asymptotic distribution under $H_{1}$ in (8), is larger for an imaging detector. Evaluation of (8) for an imaging and non-imaging detector shows that imaging capability improves the AUC if and only if

$$
\left(\mathrm{F}(\boldsymbol{\theta})_{[1,1]}^{-1}\right)^{-1} \geq \tilde{\mathrm{F}}(\boldsymbol{\theta}) .
$$

Theorem 1 shows that this inequality holds for all uniformsensitivity detectors. The proof will be given in the full version of this work [9].

Theorem 1. For a uniform sensitivity imaging detector in a known background, $\left(\mathrm{F}^{-1}(\boldsymbol{\theta})_{[1,1]}\right)^{-1}>\tilde{\mathrm{F}}(\boldsymbol{\theta})$, i.e., the reciprocal of the $[1,1]$ component of the inverse Fisher Information Matrix (9) for an imaging detector is greater than that of a non-imaging detector (10). Therefore, the asymptotic AUC for an imaging detector is greater than the asymptotic AUC of a non-imaging detector in a known background when both detectors have uniform sensitivity.

In the case where $K_{[2,1]}=\mathbf{0}$, which is typical in uniformsensitivity detectors, one can show that

$$
\left(\mathrm{F}^{-1}(\boldsymbol{\theta})_{[1,1]}\right)^{-1}=K_{[1,1]} \tilde{\mathrm{F}} .
$$

This says that imaging increases the noncentrality parameter $\eta$ in (8) by the factor $K_{[1,1]}$ in (11).

In practice, sensitivity of gamma-ray detectors is rarely uniform. When sensitivity is nonuniform, the source position becomes a nuisance parameter and can degrade detection performance. One can evaluate the Fisher information matrix (11) for a particular detector geometry to evaluate its asymptotic detection performance [9].

\section{NUMERICAL RESULTS}

In this section, we numerically evaluate the Fisher information (11) for 2D circular detectors of radius $r$ to illustrate Theorem 1, because a $2 \mathrm{D}$ circular detector has uniform sensitivity. In $2 \mathrm{D}$, we measure the far-field source position by its angle counterclockwise from the $x$-axis. In this case, the position parameter vector $\phi$ is a scalar and we denote it by $\phi$. The set of possible source positions is $\Phi=[0,2 \pi)$.

\section{A. Setup for Numerical Calculations}

For simplicity, we assume that these detectors only record single photon interaction events. For each recorded event, the detector records the position of the interaction $(x, y)$. The attribute vector $\boldsymbol{r}_{i}$ is the interaction position of the $i$ th event. Although this hypothetical detector does not necessarily represent a practical system, the attribute vector $\boldsymbol{r}$ has length 2, so one can practically compute the components of the Fisher 
information (12) numerically using the Riemann approximation

$$
\begin{aligned}
K_{[i, j]} & =\int_{\mathcal{R}} \frac{g_{i}(x, y) g_{j}^{T}(x, y)}{\mathrm{p}(x, y \mid D ; \boldsymbol{\theta})^{2}} \mathrm{p}(x, y \mid D ; \boldsymbol{\theta}) d x d y \\
& \approx \sum_{i=1}^{n_{x}} \sum_{\substack{j=1 \\
\left(x_{i}, y_{i}\right) \in \mathcal{R}}}^{n_{y}} \frac{g_{i}\left(x_{i}, y_{j}\right) g_{j}^{T}\left(x_{i}, y_{j}\right)}{\mathrm{p}\left(x_{i}, y_{j} \mid D ; \boldsymbol{\theta}\right)} \Delta_{x} \Delta_{y},
\end{aligned}
$$

where $i=1,2, \ldots, n_{x}, j=1,2, \ldots, n_{y}$, and the points $\left(x_{i}, y_{i}\right)$ are samples of $\mathcal{R}$ with uniform spacing of $\Delta_{x}$ in the $x$-direction, and $\Delta_{y}$ in the $y$-direction.

The probability density of interaction locations is governed by the Beer-Lambert law, which is also known as the attenuation law [10, pp. 54-56]. This means that the density of interaction events at a particular point inside the detector is a decreasing function of the length of material that a photon must pass through to reach that point. The number of photons, on average, that interact in a given length of material is parameterized by $\mu$, the material linear attenuation coefficient at the energy of incoming gamma-rays, which is assumed to be known. The attenuation coefficient serves as a measure of detector quality, and is related to its position resolution. The interaction probability distribution is given by

$$
\mathrm{p}(x, y \mid D ; \boldsymbol{\theta})=\frac{1}{l(\phi) \operatorname{Pr}(D)} \mu e^{-\mu d_{i}(x ; y, \phi)} \quad(x, y) \in \mathcal{R},
$$

where $d_{i}(x ; y, \phi)$ is the distance that the photon must travel along the line from the source to the position of interaction, and $l(\phi)$ is the largest distance between any two lines with slope $\tan (\phi)$ that pass through the detector, which is $2 r$ for this detector. The exact expression for $d_{i}(x ; y, \phi)$ is

$d_{i}(x ; y, \phi)=\sqrt{r^{2}-(x \sin \phi-y \cos \phi)^{2}}-(x \cos \phi+y \sin \phi)$.

One can interpret this formula as a clockwise rotation of angle $\phi$ to move the point of interaction to the $x$-axis. Then the distance from the edge of the detector is the difference between the intersection of the detector boundary with the $x$-axis and the $x$-coordinate of the interaction location. The quantities described above are illustrated in Figure 2 for the 2D circular detector.

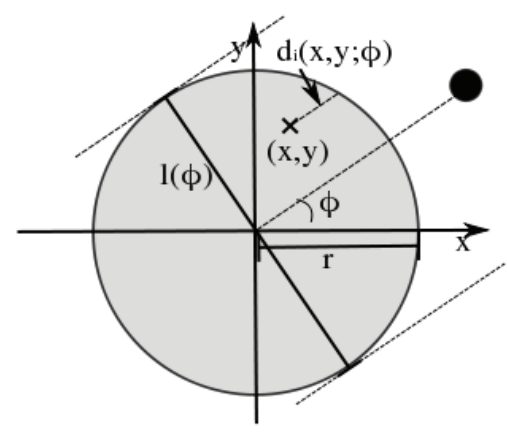

Fig. 2: Diagram of 2D circular detector.
It is difficult to define the intrinsic position resolution of these systems, so we report the angular uncertainty measured by the square root of the Cramer-Rao lower bound on the position estimate $\sqrt{\left(F(\theta)^{-1}\right)_{[2,2]}}$, where $F(\theta)$ is defined in (11).

\section{B. Results for 2D Circular Detector}

Figure 3 shows the AUC, the imaging gain factor $K_{[1,1]}$ from (11), and angular uncertainty as a function of attenuation-radius product for a circular detector. The quantity $\alpha \tau$ represents the expected number of photons emitted from the source during the scan and $\lambda_{b} \tau$ represents the expected number of background photons recorded. The expected number of source photons recorded is given by $\alpha \tau s_{0}$. As guaranteed by Theorem 1, the AUC of the imaging detector is always higher than that of the the non-imaging detector. Figure $3 \mathrm{~d}$ shows $s_{0}$ as a function of $\mu r$ to aid interpreting Figure 3a. Recall that $K_{[1,1]}$ is the multiplicative improvement in the noncentrality parameter of the asymptotic distribution of the GLRT under $H_{1}$, as expressed in (14). As $\mu r$ increases, the angular uncertainty decreases and the difference in AUC between the detectors with and without imaging information increases. Figures $3 \mathrm{a}$ and $3 \mathrm{~b}$ illustrate that as the detector provides more precise imaging information, the improvement in detection performance due to imaging information increases even when the background is known.

Figure 4 shows the AUC for a circular uniform-sensitivity detector as a function of source intensity for a fixed background intensity. The difference between the AUC for the imaging and non-imaging detector is greatest when the source-to-background ratio is near 1:1. For low source-tobackground ratios, the source is difficult to detect with either detector, and when the source-to-background ratio approaches infinity, the source is so detectable that imaging information provides little additional benefit. The detector in Figure 4 is identical to that in Figure 3d, so their sensitivities are equal. Again, the imaging detector always has higher AUC than the non-imaging detector as expressed in Theorem 1 .

\section{CONCLUSion ANd Future Work}

We investigated how imaging capability impacts the detection performance in a photon counting detector. In the case of a uniform-sensitivity detector in known background, we showed in Theorem 1 that imaging always improves detection performance in terms of asymptotic AUC.

This work focused on evaluating the detection performance of photon counting imaging detectors and comparing it to the performance of non-imaging detectors using ROC analysis. Since in addition to accurate detection, accurate localization is important in practice, future work should examine the localization ROC (LROC) of gamma-ray detectors. LROC analysis has previously been applied to study detection and localization performance in medical imaging [11]. Future work could extend this analysis to networks of imaging sensors. Since non-imaging sensors are typically much less expensive, future work will lay the foundation for cost-benefit analysis 


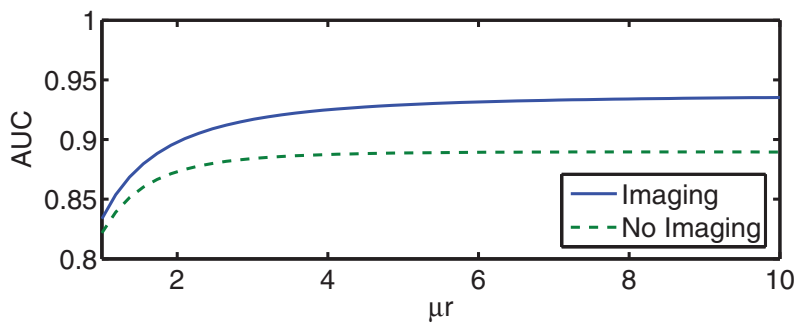

(a) AUC versus $\mu r$

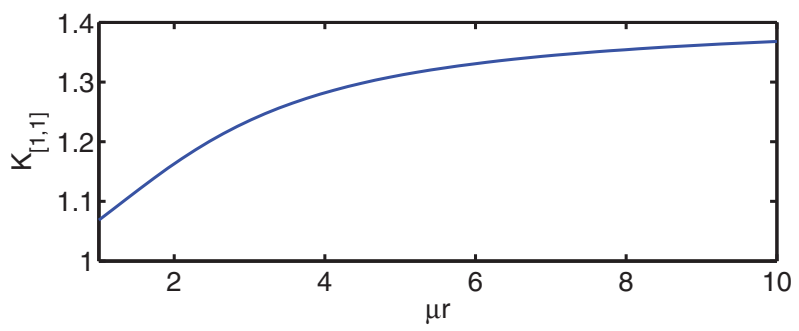

(b) $K_{[1,1]}$ versus $\mu r$

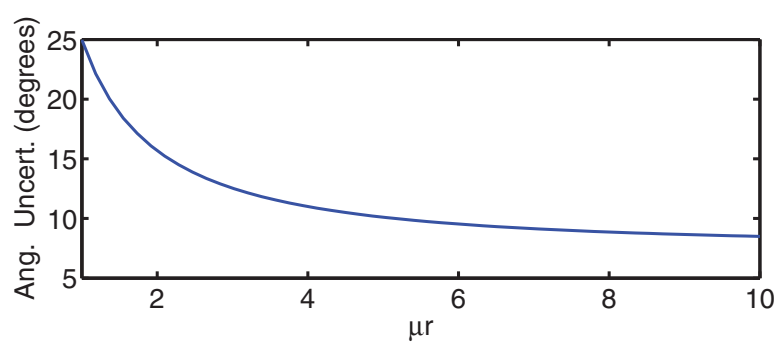

(c) Angular Uncertainty versus $\mu r$

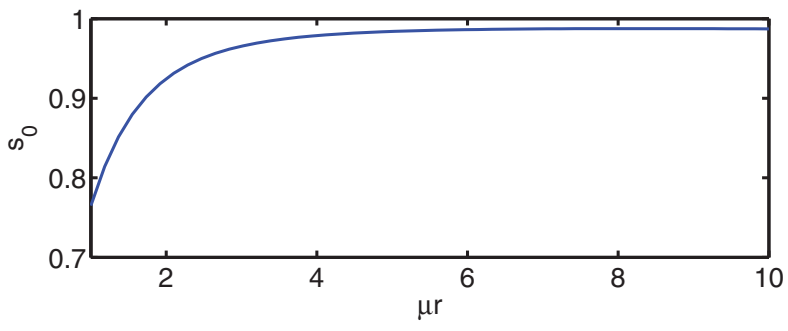

(d) $s_{0}$ versus $\mu r$

Fig. 3: Various quantities for a circular uniform-sensitivity detector with $\lambda_{b} \tau=\alpha \tau=10$.

for use of imaging detectors for networked applications. The numerical calculations considered single a photon energy, and future work should consider energy spectra [12]. The numerical results were for single photon interaction detectors, and future work would extend these results to Compton detectors, which can record multiple interactions.

\section{REFERENCES}

[1] R. C. Runkle, T. M. Mercier, K. K. Anderson, and D. K. Carlson, "Point source detection and characterization for vehicle radiation portal monitors," Nuclear Science, IEEE Transactions on, vol. 52, no. 6, pp. 3020-3025, 2005.

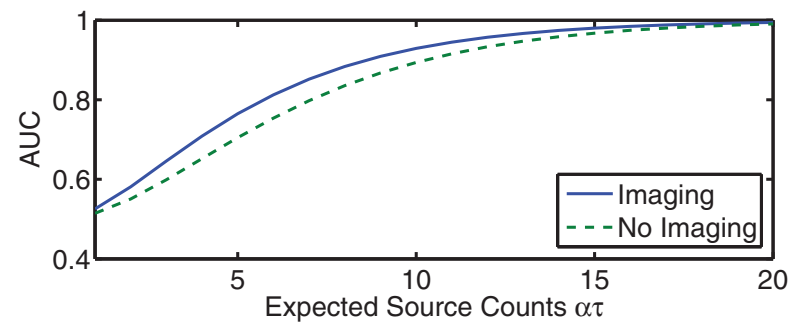

Fig. 4: AUC versus expected source counts for a circular uniform-sensitivity detector with $\lambda_{b} \tau=10$ and $\mu r=5$.

[2] C. G. Wahl and Z. He, "Sensitivity of gamma-ray source detection using 3d-position-sensitive semiconductor detectors," in Nuclear Science Symposium Conference Record, 2008. NSS 'O8. IEEE, 2008, pp. 33343338 .

[3] K. P. Ziock, W. W. Craig, L. Fabris, R. C. Lanza, S. Gallagher, B. K. P. Horn, and N. W. Madden, "Large area imaging detector for longrange, passive detection of fissile material," Nuclear Science, IEEE Transactions on, vol. 51, no. 5, pp. 2238-2244, 2004.

[4] J. A. Hanley and B. J. McNeil, "The meaning and use of the area under a receiver operating characteristic (ROC) curve." Radiology, vol. 143, no. 1, pp. 29-36, April 1982.

[5] H. H. Barrett, T. White, and L. C. Parra, "List-mode likelihood," J. Opt. Soc. Am. A, vol. 14, no. 1, pp. 2914-23, Nov. 1997.

[6] S. M. Kay, Fundamentals of statistical signal processing: Detection theory. New York: Prentice-Hall, 1998.

[7] R. Protassov, D. A. van Dyk, A. Connors, V. L. Kashyap, and A. Siemiginowska, "Statistics: Handle with care, detecting multiple model components with the likelihood ratio test," Astrophys. J., vol. 571, pp. 545-559, Jan 2002.

[8] Z. Liu and A. Nehorai, "Statistical angular resolution limit for point sources," Signal Processing, IEEE Transactions on, vol. 55, no. 11, pp. 5521-5527, 2007.

[9] D. J. Lingenfelter, J. A. Fessler, C. D. Scott, and Z. He, "Benefits of position-sensitive detectors for radioactive source detection," IEEE Trans. Sig. Proc., 2009, submitted as T-SP-08958-2009.

[10] G. F. Knoll, Radiation detection and measurement, 2nd ed. New York: Wiley, 1989.

[11] P. K. Khurd and G. R. Gindi, "LROC model observers for emission tomographic reconstruction," in Proc. SPIE 5372, Medical Imaging 2004: Image Perception, Observer Performance, and Technology Assessment, 2004, pp. 509-20.

[12] D. Xu and Z. He, "Gamma-ray energy-imaging integrated spectral deconvolution," Nucl. Instr. Meth. Phys. Res. A., vol. 574, no. 1, pp. 98-109, Apr. 2007. 